\title{
Conformational studies on two FtsZ targeting cyclic peptides
}

Nikolina Vidović, ${ }^{1 *}$ Teresa Recca, ${ }^{2}$ Pierangelo Francescato, ${ }^{1}$ Marco Rabuffetti, ${ }^{3}$ Maurizio Sironi, ${ }^{1,4}$ Francesco Oliva, ${ }^{1}$ Stefano Pieraccini, ${ }^{1,4}$ Giovanna Speranza ${ }^{1,4^{*}}$

\section{nikolina.vidovic@unimi.it; giovanna.speranza@unimi.it; +3902503 14097}

\author{
${ }^{1}$ Department of Chemistry, University of Milan, Via C. Golgi 19, 20133 Milano, Italy \\ ${ }^{2}$ Department of Chemistry, University of Pavia, Via T. Taramelli, 12, 27100 Pavia, Italy \\ ${ }^{3}$ Department of Food, Environmental and Nutritional Sciences, University of Milan, Via L. Mangiagalli, 25 - \\ 20133 Milano \\ ${ }^{4}$ Institute of Molecular Science and Technologies (ISTM-CNR), Via C. Golgi 19, 20133 Milano, Italy
}

\begin{abstract}
Two FtsZ targeting cyclic peptides 1 (Ac-[Orn-Leu-Met-Asp]-Ala-Phe-Arg-Ser-NH ${ }_{2}$ ) and 2 (Ac-Ser-Leu-Met[Asp-Ala-Phe-Arg-Orn]- $\mathrm{NH}_{2}$ ) were found to be inhibitors of FtsZ polymerization, that makes them excellent starting point for the future development of a new class of antimicrobials. We investigated their solution structure by means of nuclear magnetic resonance (NMR) and molecular dynamic simulations (MD).

Deep analysis of 2D NMR spectra (COSY, TOCSY and NOESY), recorded in DMSO- $d_{6}$, allowed the assignments of all peptide signals and suggested the presence of strong turn structures. We also noticed that the guanidine group of Arg significantly affects the spectral properties and the chromatographic behavior of these peptides depending on whether it makes part of the cycle or not. MD simulations allowed to investigate the conformational preference of the two cyclic peptides and to associate diversity in their structure and dynamics to their different behavior. In particular, peptide $\mathbf{1}$ showed enhanced flexibility and structural variance with respect to peptide 2 .
\end{abstract}

\section{KEYWORDS}

Cyclic peptides, FtsZ targeting peptides, conformation, NMR, Molecular dynamics 


\section{INTRODUCTION}

Tuberculosis is infectious disease caused by Mycobacterium tuberculosis (Ahmad 2016). Due to the fact that pathogen strains very quickly develop resistance to current antibiotics (Wang 2003), there is an urgent need of new antibacterial targets and drugs with novel mechanism of action (Payne 2008, Marcelo 2013). The tubulin homologue FtsZ, present in most prokaryotes, is considered an attractive target for the development of effective antibiotics against tuberculosis (Ahmad 2016) because of its essential role in cell division (Läppchen 2005) and its known biochemical activity (White 2000). Thus, inhibition of FtsZ polymerization would prevent cells from dividing, leading to cell death.

Sironi et el. (2013) investigated the network of interactions between two bound monomers of Mycobacterium tuberculosis FtsZ through molecular dynamics and free energy calculations. Such in silico study led to the design of two cyclic peptides 1 and $\mathbf{2}$ (Fig. 1) which were synthesized by solid phase peptide synthesis (SPPS) and investigated in vitro for their influence on FtsZ polymerization. Although both peptides were found to be active in inhibiting GTPase activity, thus affecting the FTsZ dynamycs, a detailed structural knowledge of their conformation is lacking.

In recent years, structure-based drug design (SBDD) has become a crucial method for modern drug discovery since it permits the identification and validation of drug leads and reduce the cost and times for drug research. Improvement of NMR techniques and rapid advancement in computational chemistry make a combination of these two methods extensively used to assist the development of new drugs in various stages of the SBDD process. For this reason we investigated the conformational preferences of the two bioactive cyclic peptides $\mathbf{1}$ and $\mathbf{2}$ through an integrated molecular modeling - NMR spectroscopy approach. This work reports the results of such structural analysis.

\section{RESULTS AND DISCUSSION}

The peptides 1 and $\mathbf{2}$ (Fig. 1) were synthesized by standard fluorenyl-9-methoxycarbonyl (Fmoc) solid-phase synthetic protocol on Rink Amide resin support using HOBt/HBTU as the condensing agents, purified by semipreparative RP-HPLC and checked for purity by analytical RP-HPLC as previously reported (Sironi 2013). Their identity and molecular weight were confirmed by ESI-MS (in both positive and negative modes) and MALDI TOF mass spectrometry (Fig. S1 - S3).
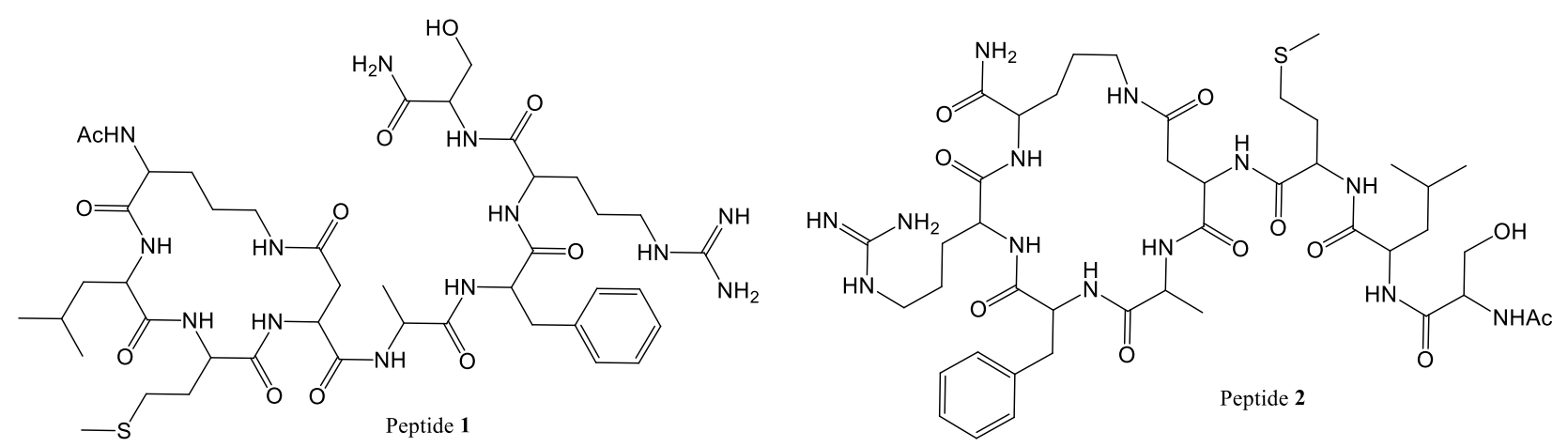

Fig. 1 Structure of peptides 1 and 2 
It is worth noting that, despite the presence of only one couple of scrambled amino acids in the sequence, the two peptides showed a very different chromatographic behavior in RP-HPLC under the conditions used for their purification and analysis [elution at $64 \%$ and $85 \%$ solvent B for $\mathbf{1}$ and $\mathbf{2}$, respectively, (see Materials and Methods and Fig. S4, Supp. Info)]. This prompt us to investigate in more detail their conformational properties by NMR and molecular modeling.

NMR spectra of peptides 1 and $\mathbf{2}$ were recorded in DMSO- $d_{6}$ at room temperature. Complete assignments of chemical shifts were made using the total correlation spectroscopy (TOCSY) NMR spectra (Table S1, Supp. Info). Fig. S5. shows $\alpha$-protons /side chains $-\mathrm{NH}$ region of the TOCSY spectra of both peptides.

In the ${ }^{1} \mathrm{H}$ NMR spectra of both peptides less intense peaks, representing no more than $10 \%$ population, can be observed. This percentage was obtained integrating the total peak areas of the major and minor populations and calculating their ratio. Such signals can be attributed to isomers arising from a racemization process in the course of the cyclization step and were not considered for our assignments. Spin system identification was accomplished using TOCSY spectra, taking into account the chemical shifts patterns reported in the literature (Wüthrich 1986).

It is known that the NMR-based solution structure determination of small peptides is challenging due to fast interconversion among various conformers (Ramirez 2019, Montelione 1989 ). In Fig. 2 the deviation of the observed chemical shifts of the $\alpha$-protons from random coil values (Wishart 1992) for proteinogenic amino acids is reported. Chemical shift index (CSI) calculations are commonly used to detect secondary structure elements (Schwarzinger 2001, Wishart 1992). In particular, for $\alpha$-protons, negative differences (upfield shifts) suggest the presence of a helix or turn, whereas positive differences (downfield shifts) are indicative of extended conformations (corresponding to ß-strands/ß-sheets). The chemical shifts of $\mathrm{NH}$ protons are also linked to the secondary structure, but their changes are not so significant as for $\mathrm{C} \alpha$. In addition, NH proton chemical shifts are significantly dependent on the solvent and temperature and for this reason we didn't take them into consideration.

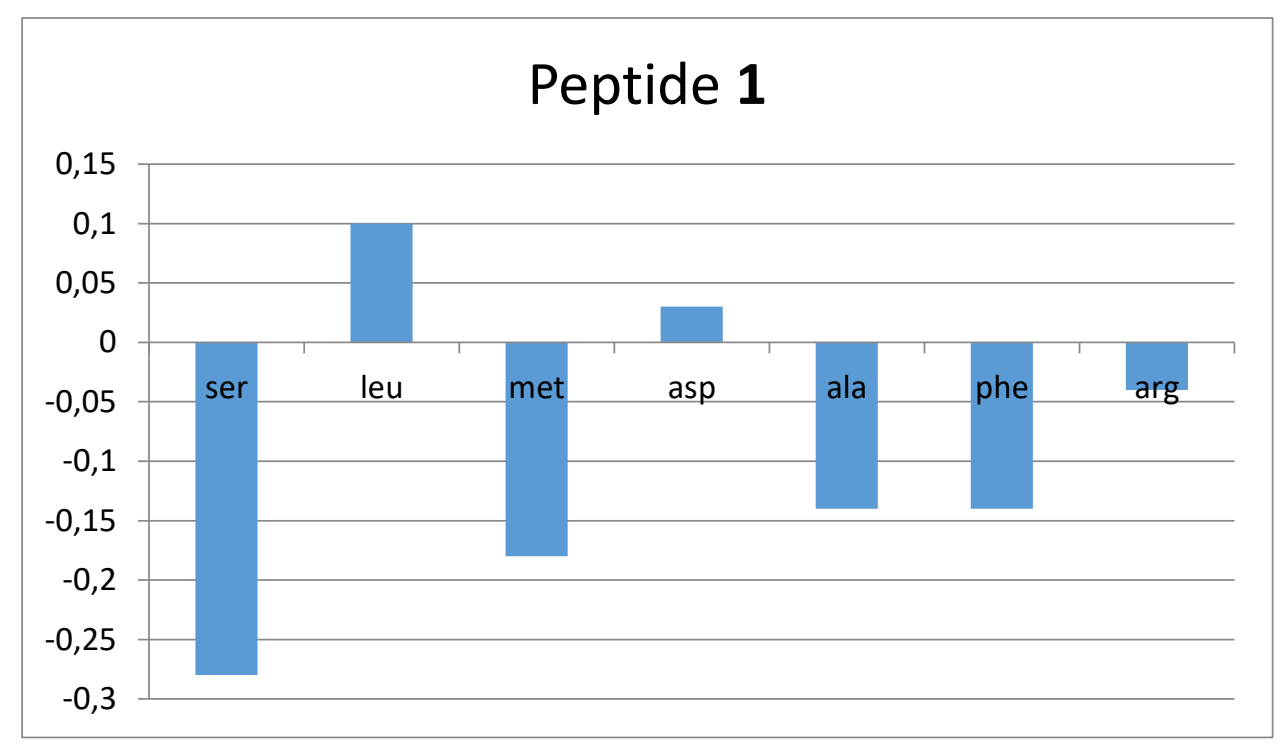




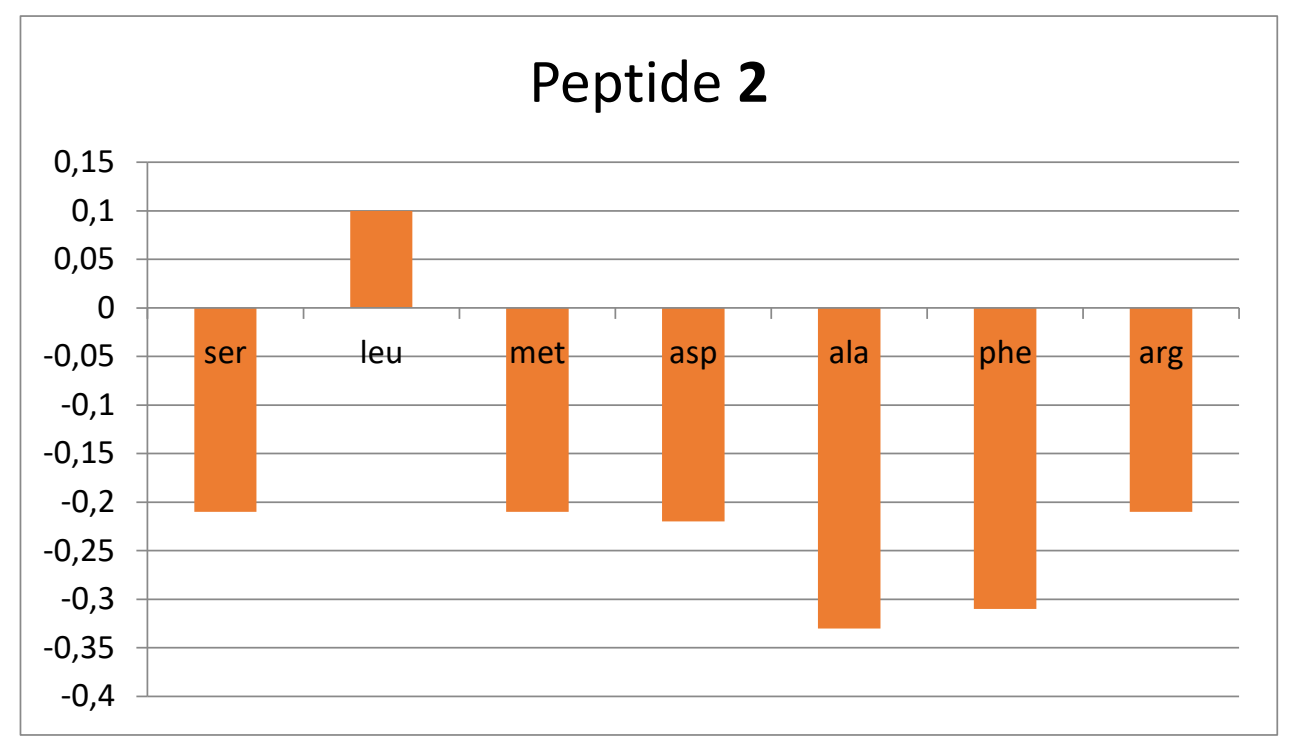

Fig. 2 Chemical shifts of the $\alpha$-protons of peptides $\mathbf{1}$ and $\mathbf{2}$ from random coil values

Analysis of CSI indicates that for all residues the deviations from the random coil follow the same trend except for Asp which has a very small positive value $(0.03)$ in peptide $\mathbf{1}$ and a negative value $(-0.22)$ in peptide $\mathbf{2}$ suggesting that a well-ordered conformation may exist around this residue. In addition, in both peptides Leu show positive difference (0.1), being negative the differences for the remaining residues. In peptide $\mathbf{2}$, the deviation from random coil is greater for Ala and Phe, the residues close to Asp and part of the cycle, with respect to other amino acids. By contrast, in peptide $\mathbf{1}$ the highest deviation value is found for the $C$-terminal capped residue, Ser, while for the residues inside the cycle, Leu and Met, opposite deviations are observed. As for Arg, the difference is close to zero (-0.04) in peptide 1 and significantly negative for peptide $\mathbf{2}(-0.21)$ indicating that Arg in peptide $\mathbf{1}$ is highly dynamic with no secondary structural preference while in peptide $\mathbf{2}$ has a limited conformational freedom. These results are in a good agreement with those obtained by MD simulations. Considered all this, for both peptides the observed deviations from random coil values suggest a preference for organized rather than random conformations.

The notable difference in the chemical shift of Arg peaks between $\mathbf{1}$ and $\mathbf{2}$ can be attributed to the effect of the different interactions in solution of the guanidine groups in the two peptides. In fact, in peptide $\mathbf{2} \operatorname{Arg}$ is part of the cycle and interactions of its side chain with the remaining cycle constituents are detectable (see below). By contrast, in peptide 1 guanidine group is out of the cycle and more prone to interactions and formation of hydrogen bonds with the solvent (Burgess 1995).

Nuclear Overhauser effect (NOEs) is the most important source of structural information to define the peptide geometry due to the dependence of the cross peak intensity on the distance between nuclei. Both short range $[d N N(i, i+1)]$ and $[d \alpha N(i, i+1),[d \alpha N(i, i+2)$ and $d \alpha N(i, i+3)]$ NOEs are observed when the peptide has a significant population of turns or helices. 

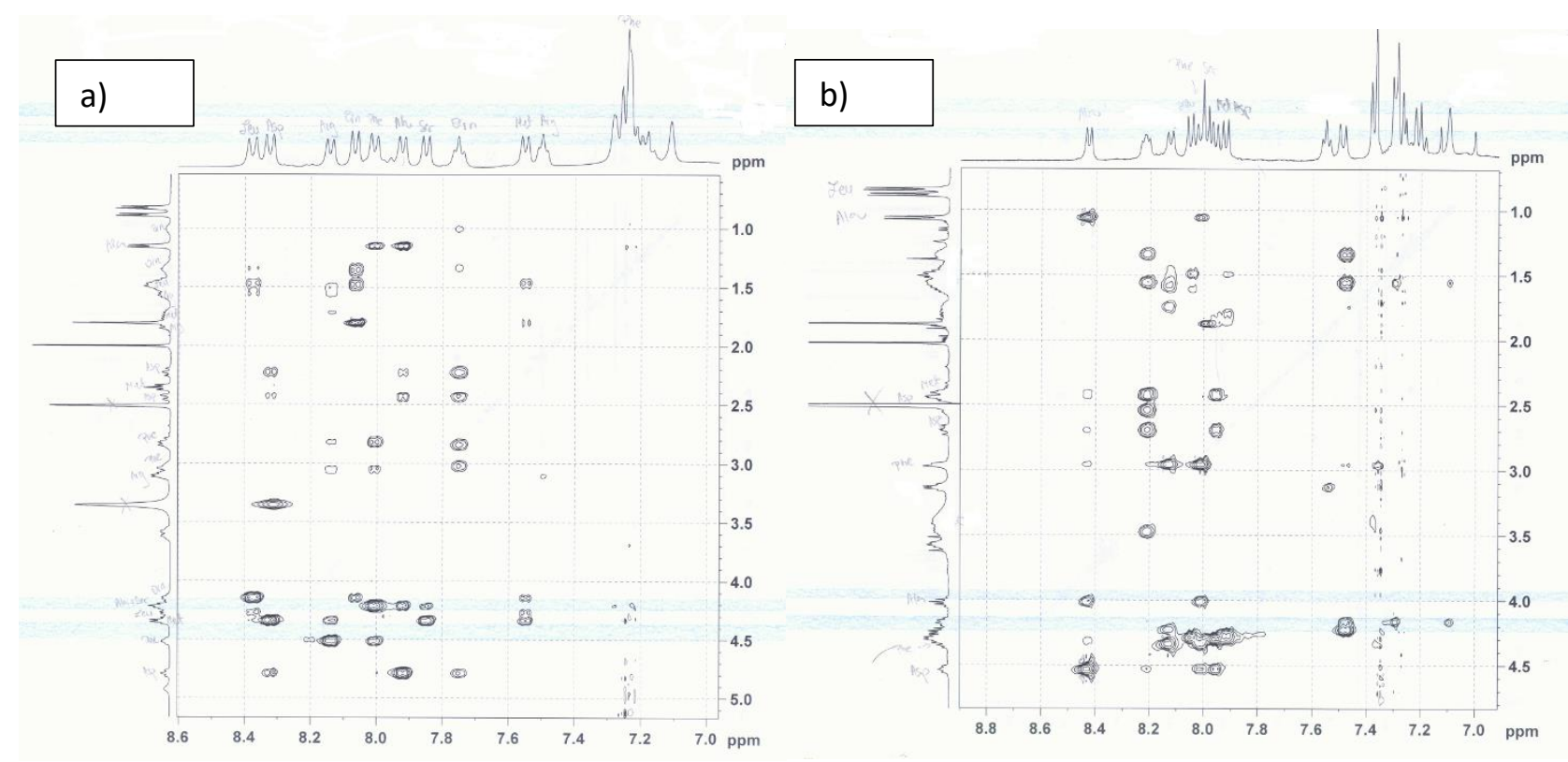

Fig. 3 Portions of NOESY spectra of peptide $\mathbf{1}(a)$ and $\mathbf{2}(b)$

The aliphatic-NH region of the 2D NOESY spectrum of the peptide 1 and the complete list of the observed NOE signals are reported in Fig. 3a and in Table S3 (Supp. Info), respectively. The presence of all sequential short range $d N N(i, i+1)$ NOEs of amino acids within the cycle as well as of all expected $d \alpha N(i, i+1)$ and some $\mathrm{d} \alpha \mathrm{N}(\mathrm{i}, \mathrm{i}+2)$ NOE cross-peaks is indicative of a turn structure.

Ala, that is the first amino acid outside the ring, shows strong interaction with Asp, while for the three remaining residues out of the cycle (Phe, Arg and Ser) it was not possible to attribute sequential dNN(i,i+1) NOE connectivities but only strong sequential $d \alpha N(i, i+1)$ NOEs are observed. Such a result is indicative of a well-defined conformation (Type I $\beta$-turn) (Desai 2002) for this segment of the molecule in agreement with previous studies showing that homochiral sequences (Brenner 2007) as well as those containing Asp predominantly accommodate Type I $\beta$-turn (Mukrasch 2007). The absence of any interaction of the guanidine group NH protons of the arginine residue with the other residues suggests that Arg side chain is in totally free motion. By contrast, a strong interaction of Ha protons of Asp with NH protons of Orn's side chain occurs. Fig. $3 \mathrm{~b}$ shows the aliphatic-HN region of the 2D NOESY spectrum of the peptide 2 . It is worth noting that $\mathrm{NH}$ protons of Ser interact with $\operatorname{Arg}$ ( $\beta$ and $\gamma$ hydrogens), Ala ( $\alpha$ and $\beta$ hydrogens) and Asp ( $\alpha \mathrm{H}$ ) suggesting the formation of a strong turn structure. Side chain NH protons of $\mathrm{Arg}$, at the same time, show contacts with $\alpha$ and $\beta$ protons of Asp as well as with $\mathrm{NH}$ protons of Ser. Even in this case the presence of the interactions of Ha protons of Asp with NH protons of Orn's side chain is observed.

Scalar coupling constants were calculated using the backbone amide region of the ${ }^{1} \mathrm{H}$ NMR spectrum (for the complete list of $\mathrm{J}^{3}$ values see Table S4, Supp. Info). The ${ }^{3} \mathrm{~J}_{\mathrm{HN}-\alpha \mathrm{H}}$ coupling constants is a valuable NMR parameter to distinguish the secondary structure in proteins. For small peptides, the backbone coupling constants are usually around rotationally averaged values $(7 \mathrm{~Hz}$ ) (Dyson 1991). Thus, values between 6 and $8 \mathrm{~Hz}$ are usually not included in the structure calculations. For peptide 1 large value of coupling constants for Leu and Asp ( 9.4 and $8.8 \mathrm{~Hz}$, respectively) suggested an ordered structure. For peptide $\mathbf{2}$, only coupling constant of serine has a higher value $(8.7 \mathrm{~Hz})$ than the conformational average indicating well-defined local structure around this residue. 
In order to get an atomic level insight into the preferred conformations of peptides $\mathbf{1}$ and $\mathbf{2}$ in solution, molecular dynamics simulations in explicit DMSO solvent were performed. DMSO was chosen in order to reproduce the conditions of NMR spectra registration. To have a general idea of the conformational preferences of the two peptides, a cluster analysis on each of the MD trajectories was performed, based on the root mean square distance matrix of heavy atoms. In this way, it has been possible to outline the different families of conformations (clusters) assumed by each peptide. Two threshold values were employed for clustering, $0.1 \mathrm{~nm}$ and $0.2 \mathrm{~nm}$ respectively, corresponding to a fine discrimination between distinct structural families and to a more coarse grain evaluation. The gromos clustering method (Daura 1999) has been employed. The results are shown in Tables 1, 2, 3 and 4.

Table 1 Peptide 1 cluster analysis with cut-off of $0.1 \mathrm{~nm}$

\begin{tabular}{lll}
\hline \multicolumn{1}{c}{ cutoff 0.1} \\
\hline Number of clusters & Population first 5 clusters & Cluster's Weight (\%) \\
75 & 798 & 15.96 \\
& 613 & 12.26 \\
& 405 & 8.10 \\
& 403 & 8.06 \\
& 272 & 5.44 \\
\hline
\end{tabular}

Table 2. Peptide 1 cluster analysis with cut-off of $0.2 \mathrm{~nm}$.

\begin{tabular}{lll}
\hline \multicolumn{2}{c}{ cutoff $\mathbf{0 . 2}$} \\
\hline Number of clusters & Population first 5 clusters & Cluster's Weight (\%) \\
\hline 4 & 3060 & 61.19 \\
& 1633 & 32.65 \\
& 286 & 5.72 \\
& 22 & 0.44 \\
\hline
\end{tabular}

Table 3. Peptide 2 cluster analysis with cut-off of $0.1 \mathrm{~nm}$.

\begin{tabular}{lll}
\hline & \multicolumn{1}{c}{ cutoff $\mathbf{0 . 1}$} \\
\hline Number of clusters & Population first 5 clusters & Cluster's Weight (\%) \\
\hline 32 & 2307 & 46.13 \\
& 444 & 8.88 \\
& 383 & 7.66 \\
& 348 & 6.96 \\
& 338 & 6.76 \\
\hline
\end{tabular}

Table 4. Peptide $\mathbf{2}$ cluster analysis with cut-off of $0.2 \mathrm{~nm}$.

\begin{tabular}{lll}
\hline \multicolumn{2}{c}{ cutoff $\mathbf{0 . 2}$} \\
\hline Number of clusters & Population first 5 clusters & Cluster's Weight (\%) \\
3 & 4541 & 90.80 \\
& 367 & 7.34 \\
& 30 & 0.60 \\
\hline
\end{tabular}


Concerning the finer clustering, corresponding to the $0.1 \mathrm{~nm}$ threshold, we observed that peptide 2 shows a fairly smaller number of clusters with respect to peptide 1 (32 vs 75), indicative of a more restrained conformational space associated to a more rigid structure. The same tendency is observed with the $0.2 \mathrm{~nm}$ threshold. In particular, it is worth noting that the dominant cluster of peptide $\mathbf{2}$ in this case comprises more than $90 \%$ of the structures observed during the simulation, while for peptide $\mathbf{1}$ it comprises slightly more than $60 \%$ of the structures. These results are in agreement with the CSI analysis.

To get a further insight into the structural features of peptides $\mathbf{1}$ and $\mathbf{2}$, we considered the central structure of the most populated clusters obtained with a cutoff of $0.2 \mathrm{~nm}$ (Fig. 4). Such structures are those exhibiting the smaller average distance with respect to all the members of their cluster and can be considered as representative of its most relevant structural characters.
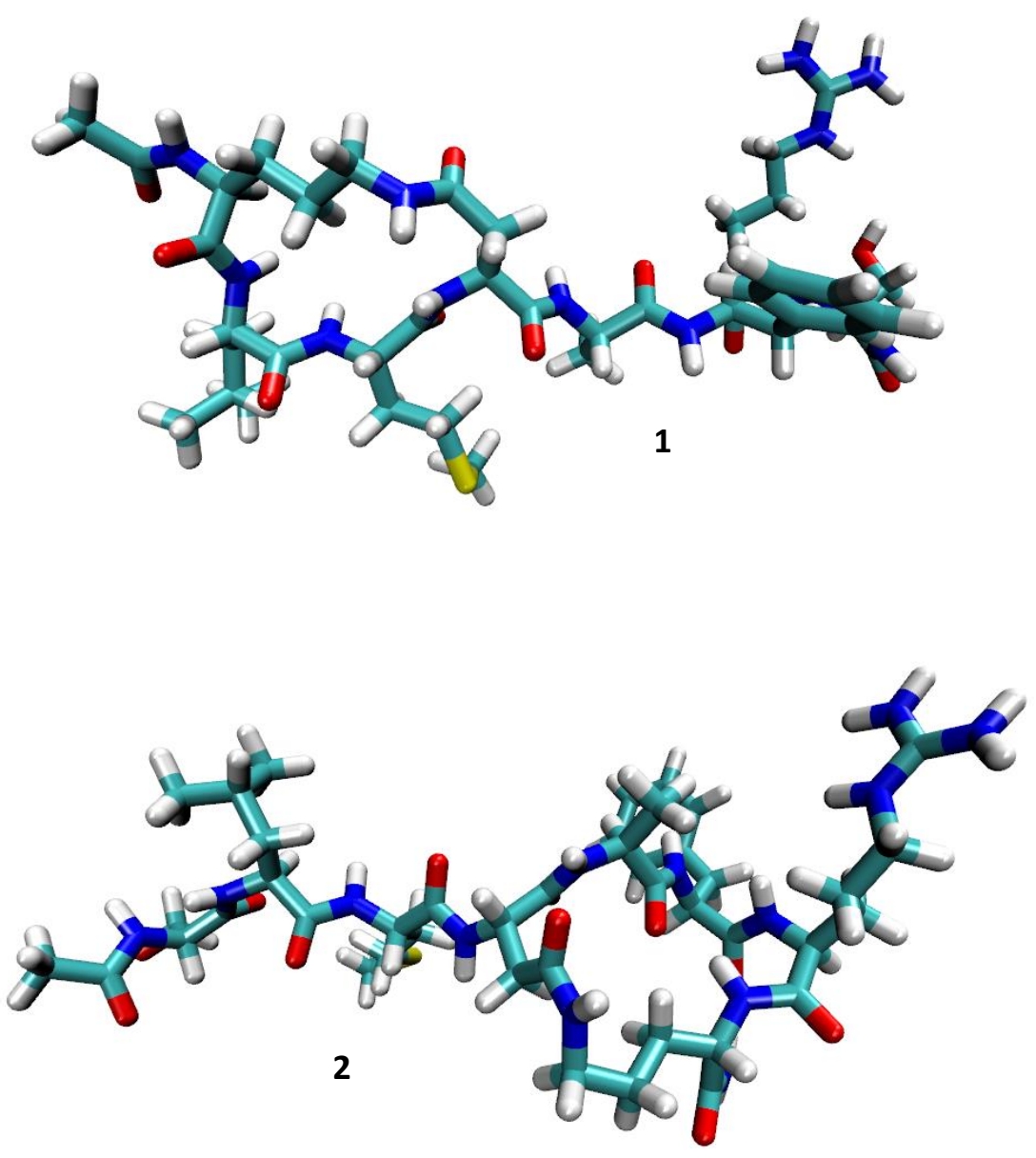

Fig. 4 Central structure of the most populated clusters. Peptide $\mathbf{1}$ (up) and peptide $\mathbf{2}$ (down)

The identification of secondary structure elements on short peptides comprising a cyclic moiety is somehow problematic. Anyway, 2D Ramachandran plots derived from the central structure of the principal clusters (Fig. 5) show a limited propensity for helical conformation in residues Phe, Arg and Orn belonging to the cyclic moiety in peptide 2 while the remaining residues (Met, Asp, Leu, Ser and Ala) show a $\beta$-sheet like conformation. Peptide 1 on the other hand shows Ser, Arg and Leu residues in helical conformation while Met, Ala, Asp, Orn and Phe assume a $\beta$-sheet conformation. The 3D Ramachandran plots (Fig. S6, Supp. Info) give a further pictorial representation of the limited conformational variability of peptide $\mathbf{2}$ in comparison to 
peptide 1. Analysis of the per residue root mean square fluctuation (RMSF) shows a significantly reduced RMSF value for Arg in peptide 2 with respect to peptide $1(0.16 \mathrm{~nm}$ vs $0.31 \mathrm{~nm})$, clearly indicating its lower flexibility when comprised into the cycle, in agreement with NMR data. A parallel, slight reduction in the average number of Arg -solvent hydrogen bonds per simulation snapshot is also observed ( $0.53 \mathrm{vs} 0.57 \mathrm{HB}$ ).
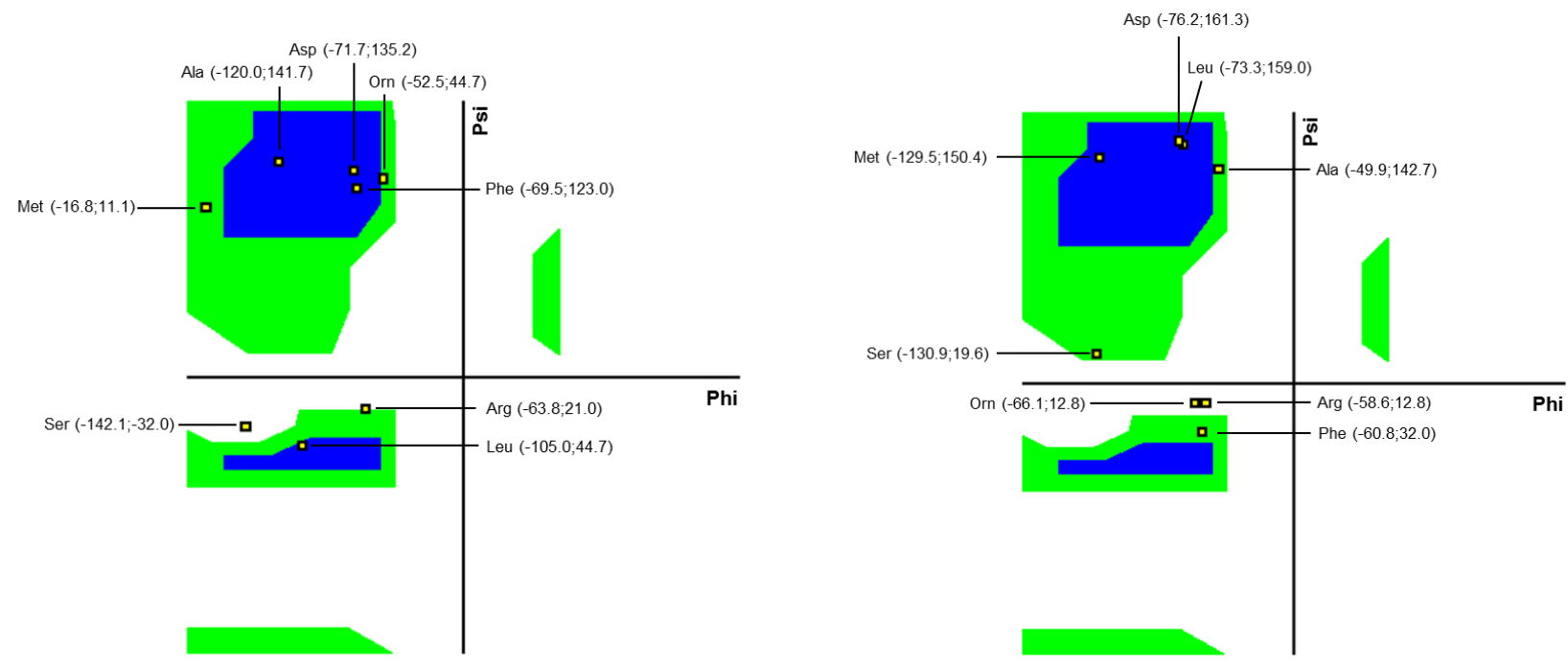

Fig. 5 Ramachandran plot. Peptide 1 on the left and peptide $\mathbf{2}$ on the right

\section{CONCLUSIONS}

The solution structure of two FtZ targeting cyclic peptides ( $\mathbf{1}$ and $\mathbf{2}$ ) was investigated using an integrated MD - NMR approach. Both peptides, which differ only for a scrambled residue, show a defined structures with residues inside the cycles adopting turn conformations. Differences in the chromatographic and spectral properties of $\mathbf{1}$ and $\mathbf{2}$ are mainly attributable to the position of the Arg residue along the sequence. Indeed, the guanidine group is rigid when Arg makes part of the cycle as in $\mathbf{2}$ while it is more prone to interactions with the surrounding molecules when Arg is outside the cycle as in $\mathbf{1}$. Both peptides were found to inhibit FtsZ polymerization and can be considered excellent candidates for development of therapeutic agents against bacterial proliferation.

\section{MATERIALS AND METHODS}

NMR experiments were performed at $298 \mathrm{~K}$ on $400 \mathrm{MHz}$ Bruker NMR spectrometer (Bruker Corporation, Billerica, MA, USA) equipped with a z-gradient coil probe. All NMR samples were prepared by dissolving lyophilized peptides in DMSO- $d_{6}$ with a peptide concentration of ca. $20 \mathrm{mg} / \mathrm{mL}$. Chemical shifts $(\delta)$ are given in parts per million and were referenced to the solvent signal $\left(\delta \mathrm{H} 2.50 \mathrm{ppm}\right.$ for DMSO- $\left.d_{6}\right)$. All 1D and 2D NMR spectra were collected using the standard pulse sequences available with Bruker Topspin 1.3. 2D TOCSY and 2D NOESY were recorded with short mixing times: $60 \mathrm{~ms}$ and $600 \mathrm{~ms}$ respectively. Proton resonances were assigned using standard methods.

Matrix assisted laser desorption/ionization spectra (MALDI-TOF) were acquired on a Bruker Microflex LT Spectrometer. 
Electrospray ionization mass spectra (ESI-MS) were recorded on a Thermo Finnigan LCQ Advantage spectrometer (Hemel Hempstead, Hertfordshire, UK).

HPLC were performed using an Amersham Pharmacia Biotech (P900) liquid chromatographer connected to an UV-VIS detector; chromatographic conditions were set as follows: column for analytical HPLC, Jupiter RP18 (10 $\mu \mathrm{m}$ proteo 90A size: 250x4.60 mm, Phenomenex); column for semipreparative HPLC, Jupiter RP-18 (10 $\mu \mathrm{m}$ proteo 90A size: 250x10 mm, Phenomenex); detector $\lambda 226$ and $280 \mathrm{~nm}$; mobile phase: A (0.1 \% TFA $\left./ \mathrm{H}_{2} \mathrm{O}\right)$ and $\mathrm{B}\left(\mathrm{CH}_{3} \mathrm{CN}\right)$ gradient elution from $5 \%$ to $100 \% \mathrm{~B}$ in $60 \mathrm{~min}$.

Molecular modeling. Peptide $\mathbf{1}$ and $\mathbf{2}$ were modeled in explicit solvent with periodic boundary conditions using molecular dynamics (MD) simulations. The peptides where solvated in a box of $\sim 80 \mathrm{~nm}^{3}$ with DMSO to reproduce the NMR experiment conditions. Every system has been submitted to geometry optimization with the steepest descent algorithm with a convergence of $100 \mathrm{~kJ} \mathrm{~mol}^{-1} \mathrm{~nm}^{-1}$. Then a $1 \mathrm{~ns}$ equilibration in NVT conditions at $300 \mathrm{~K}$ was performed, followed by a $1 \mathrm{~ns}$ NPT equilibration at the 1 bar and at the same temperature. After the equilibration phase, a $100 \mathrm{~ns}$ MD simulation was run, at a reference temperature of $300 \mathrm{~K}$. Peptides 1 and 2 have been described using the Amber99SB-ILDN Force Field (Hornak 2006) and parameterized according to standard procedures as explained in reference (Gandini 2018). During the simulations temperature was held constant with the v-rescale algorithm (Bussi 2007) while pressure was kept constant through the Berendsen barostat (Berendsen 1984). MD simulations were performed using the leapfrog algorithm with $2 \mathrm{fs}$ time-step, with holonomic constraints on every bond enforced using the LINCS algorithm. Simulations and subsequent analysis were performed with the GROMACS 5.0.4 (Van Der Spoel 2005) program suite.

\section{Acknowledgements}

\section{Compliance with Ethical Standards}

Conflict of interest Authors declare that they have no conflict of interest.

Research involving Human and Animal Rights This article does not contain any study with human or animals performed by any of the authors.

\section{REFERENCES}

Ahmad F, Chandrul KK, Naz H, Tandan N (2016) Designing of Novel Inhibitors of Mycobacterium Tuberculosis H37Rv by Pharmacophore based Drug Designing and its Evaluation. Int J Curr Res Aca Rev 4(9): 5970.

Berendsen HJ, Postma JV, van Gunsteren WF, DiNola ARHJ, Haak JR (1984) Molecular dynamics with coupling to an external bath. The Journal of chemical physics 81(8): 3684-3690.

Brenner V, Piuzzi F, Dimicoli I, Tardivel B, Mons M (2007) Chirality-Controlled Formation of $\beta$-Turn Secondary Structures in Short Peptide Chains: Gas-Phase Experiment versus Quantum Chemistry. Angew Chem Int Ed 46: 2463-2466. 
Burgess K, Ho KK, Pettitt BM (1995) Conformational Effects of Substituting Methionine with (2S, 3S)-2,3Methanomethionine in Phe-Met-Arg-Phe-NH 2 . J Am Chem Soc 117: 54-65.

Bussi G, Donadio D, Parrinello M (2007) Canonical sampling through velocity rescaling. The Journal of chemical physics 126(1): 014101.

Daura X, Gademann K, Jaun B, Seebach D, Van Gunsteren WF, Mark AE (1999) Peptide folding: when simulation meets experiment. Angewandte Chemie International Edition 38(1-2): 236-240.

Desai P, Prachand M, Coutinho E, Saran A, Bodi J, Sülli-Vargha H (2002) Activity and conformation of a cyclic heptapeptide possessing the message sequence His-Phe-Arg-Trp of a-melanotropin. International Journal of Biological Macromolecules 30: 187-195.

Dyson HJ, Wright PE (1991) Defining solution conformations of small linear peptides. Annu Rev Biophys Chem 20: 519-538.

Frisch MJ, Trucks GW, Schlegel HB, Scuseria GE, Robb MA, Cheeseman JR et al (2016) Gaussian 16. Revision A, 3.

Gandini E, Dapiaggi F, Oliva F, Pieraccini S, Sironi M (2018) Well-Tempered MetaDynamics based method to evaluate universal peptidomimetics. Chemical Physics Letters 706: 729-735.

Hornak V, Abel R, Okur A, Strockbine B, Roitberg A, Simmerling C (2006) Comparison of multiple Amber force fields and development of improved protein backbone parameters. Proteins: Structure, Function, and Bioinformatics 65(3): 712-725.

Krishnan RBJS, Binkley JS, Seeger R, Pople JA (1980) Self-consistent molecular orbital methods. XX. A basis set for correlated wave functions. The Journal of Chemical Physics 72(1): 650-654.

Läppchen T, Hartog Aloysius AF, Pinas VA, Koomen GJ, den Blaauwen T (2005) GTP Analogue Inhibits Polymerization and GTPase Activity of the Bacterial Protein FtsZ without Affecting Its Eukaryotic Homologue Tubulin. Biochemistry 4: 7879-7884.

Marcelo F, Huecas S et al (2013) Interactions of Bacterial Cell Division Protein FtsZ with C8-Substituted Guanine Nucleotide Inhibitors. A Combined NMR, Biochemical and Molecular Modeling Perspective. J. Am. Chem. Soc JACS 135: 16418-16428.

Montelione G, Winkler ME, Rauenbuehler P, Wagner G, (1989) Accurate Measurements of Long-Range Heteronuclear Coupling Constants from Homonuclear 2D NMR Spectra of Isotope-Enriched Proteins. Journal Of Magnetic Resonance 82: 198-204.

Mukrasch MD, Markwick P, Biernat J, von Bergen M, Bernado' P, Griesinger C, Mandelkow E, Zweckstetter M, Blackledge M (2007) Highly Populated Turn Conformations in Natively Unfolded Tau Protein Identified from Residual Dipolar Couplings and Molecular Simulation. J Am Chem Soc 129: 5235-5243.

Payne DJ et al (2008) Desperately seeking new antibiotics. Science 321:1644-1645.

Pieraccini S, Rendine S, Jobichen C, Domadia P, Sivaraman J, Francescato P, Speranza g, Sironi M (2013) Computer aided design of FtsZ targeting oligopeptides. RSC Advances 3: 1739-1743.

Ramirez LS, Pande J, Shekhtman A (2019) Helical Structure of Recombinant Melittin. J Phys Chem B 123: 356-368.

Schwarzinger S, Kroon GJA, Foss TR, Chung J, Wright PE, Dyson HJ (2001) Sequence-Dependent Correction of Random Coil NMR Chemical Shifts. J Am Chem Soc 123: 2970-2978.

Van Der Spoel D, Lindahl E, Hess B, Groenhof G, Mark AE, Berendsen HJ (2005). GROMACS: fast, flexible, and free. Journal of computational chemistry 26(16): 1701-1718.

Wang J, Galgoci A et al (2003) Discovery of a Small Molecule That Inhibits Cell Division by Blocking FtsZ, a Novel Therapeutic Target of Antibiotics. The journal of Biological Chemistry 278(45): 44424-44428.

White EL, Ross L, Reynolds RC, Seitz LE, Moore GD, Borhani GW (2000) Slow Polymerization of Mycobacterium tuberculosis FtsZ. Journal Of Bacteriology 182(14): 4028-4034. 
Wishart DS, Sykes BD, Richards FM (1992) The chemical shift index: a fast and simple method for the assignment of protein secondary structure through NMR spectroscopy. Biochemistry 31 (6): 16471651.

Wüthrich K (1986) NMR of proteins and nucleic acids. J. Wiley \&Sons, NY.

Zhang L, Mallik B, Morikis D (2008) Structural Study of Ac-Phe-[Orn-Pro-dCha-Trp-Arg], a Potent C5a Receptor Antagonist, by NMR. Peptide Sci 90(6): 803-815. 